\title{
Interkulturelle Kompetenz als Herausforderung für das Lehramtsstudium
}

\section{Annegret Thiem}

\begin{abstract}
Zusammenfassung
Die Vorgaben der Fremdsprachenlehrpläne NRW stellen neben der funktionalen kommunikativen Kompetenz vor allem die interkulturelle Kompetenz in den Fokus der Unterrichtsziele, welche als Studieninhalt vorausgesetzt wird. Der Artikel geht der Frage nach, was genau interkulturelle Kompetenz von Studierenden erfordert und inwieweit diejenigen, die Lehramt Spanisch studieren, überhaupt auf diese Herausforderungen vorbereitet werden bzw. welche Möglichkeiten im Rahmen des Studiums gegeben sind, um Studierende zu bewusst „interkulturell Handelnden“ auszubilden.
\end{abstract}

Aber das Eigene muß so gut gelernt sein wie das Fremde. (Hölderlin)

Der neue Kernlernplan für die Sekundarstufe II Gymnasium / Gesamtschule in Nordrhein-Westfalen für das Fach Spanisch, der im Jahr 2013 verabschiedet wurde und im Herbst 2014 in Kraft getreten ist, sieht vor, funktionale und interkulturelle kommunikative Kompetenzen unter dem Leitziel der interkulturellen Handlungsfähigkeit zu vermitteln, wie das im Lehrplan übernommene Schema der Bildungsstandards für die fortgeführte Fremdsprache (Englisch / Französisch) für die Allgemeine Hochschulreife zeigt (Kernlehrplan 2013: 15f.): ${ }^{1}$

Aus diesem „Zusammenspiel“ (ibid.: 16) der Sprachlernkompetenzen wird das Ziel der Bildungsforderungen, sich ein „angemessenes und erfolgreiches Handeln im privaten wie beruflichen Leben" (ibid.: 10) anzueignen formuliert. Die Persönlichkeitsmerkmale im ersten Feld werden dabei mithilfe funktionaler Kompetenzen erworben bzw. gefestigt und stehen in unmittelbarem Zusammenhang mit der Sprachbewusstheit als zentraler Kategorie:

\footnotetext{
${ }^{1}$ Die Übernahme von Modellen aus anderen Fremdsprachen für das Verstehen der spanischen Sprache(n) und Kultur(en) ist ein gängiges Mittel. Es fehlt an vielen Stellen eine dezidiert fachinterne Perspektive.
} 


\begin{tabular}{|c|c|c|c|}
\hline \multirow{6}{*}{ Sprachlernkompetenz } & \multicolumn{2}{|c|}{ Interkulturelle kommunikative Kompetenz } & \multirow{6}{*}{ Sprachbewusstheit } \\
\hline & Verstehen & \multirow{2}{*}{$\begin{array}{l}\text { Handeln } \\
\text { Bewusstheit }\end{array}$} & \\
\hline & Wissen Einstellungen & & \\
\hline & \multicolumn{2}{|c|}{$\begin{array}{l}\text { Funktionale kommunikative Kompetenz } \\
\text { Hör-/Hörsehverstehen } \\
\text { Leseverstehen } \\
\text { Schreiben } \\
\text { Sprechen } \\
\text { Sprachmittlung } \\
\text { Verfügen über sprachliche Mittel und kommunikative Strategien }\end{array}$} & \\
\hline & \multicolumn{2}{|c|}{ Text- und Medienkompetenz } & \\
\hline & mündlich schriftlich & medial & \\
\hline
\end{tabular}

Language Awareness ist eine zusammenfassende Bezeichnung für verschiedene Ansätze zum konstruktiven, positiven und bewussten Umgang mit Mehrsprachigkeit. [...] [Es] soll ein grundsätzliches Interesse für Sprachen, sprachliche Phänomene geweckt und eine Sensibilisierung für Erscheinungen in der eigenen und der fremden Sprache erzeugt werden. (Budde 2012: 41).

Die Basis für diese Struktur des Kernlehrplans ist ein postulierter „Paradigmenwechsel von der Input- zur Outputorientierung“ (Kernlehrplan 2013: 9):

Der Output von Bildungssystemen umfasst [... ] den Aufbau von Kompetenzen, Qualifikationen, Wissensstrukturen, Einstellungen, Überzeugungen, Werthaltungen - also von Persönlichkeitsmerkmalen bei den Schülerinnen und Schülern, [...]. Schule hat nicht nur die Funktion, individuelle Leistungsfähigkeit sicherzustellen, sondern sie dient auch der kulturellen Selbstverständigung und dem sozialen Zusammenhalt der Gesellschaft. (BMBF 2009: 12).

Soweit die Theorie. In einer Welt der Globalisierung und Pluralität sind die Anforderungen an eine heterogene und plurale Gesellschaft andere, als sie einstmals in recht überschaubaren Zusammenhängen waren. Angesichts gesellschaftlicher Heterogenität, die uns im alltäglichen Leben ebenso wie in der Schule und an den Universitäten begegnet, stellen kulturelle Selbstverständigung ebenso wie ein sozialer Gesellschaftszusammenhalt die Herausforderungen unserer Zeit dar. Im Verständnis der interkulturellen Pädagogik meint Heterogenität die „Unterschiedlichkeit von Lebenslagen“ (Gogolin/Krüger-Potratz 2006: 12), welche die Grundlage ihrer Forschungsarbeit bildet und in welcher sie darum bemüht sind, „Lebens- und Bildungschancen der Heranwachsenden [... ] von den Zufällen ihrer Herkunft unabhängig“ (ibid.) zu halten, und ihnen ein Instrumentarium an die Hand zu geben, das ihnen hilft, sich ,in der sozial, kulturell und sprachlich heterogenen Gesellschaft" (ibid.: 13) zurechtzufinden. Vom Begriff 
der Heterogenität leitet sich in der Diskussion die Frage nach Interkulturalität ab. Interkulturalität meint in diesem Kontext ein Miteinander von sich voneinander unterscheidenden gesellschaftlichen Aspekten eines Landes. Das aber ist etwas anderes, als wir es als Fremdsprachenlernziel postulieren, nämlich als „Auseinandersetzung mit anderen Lebenswirklichkeiten“ (Kernlehrplan 2013: 10). Und damit haben wir schon zwei Facetten der Anwendung von Interkulturalität: Zum einen das Erlernen einer Fremdsprache als Auseinandersetzung mit einer bestimmten Zielkultur und zum anderen die Tatsache, dass es angesichts der Heterogenität unmöglich ist, von einer einheitlichen Perspektive auf diese Zielkultur zu schauen, ganz abgesehen davon, dass die Zielkultur auch nicht einheitlich sein kann, wenn wir Globalität als universales Phänomen verstehen. ${ }^{2}$ Interkulturalität, interkulturelles Lernen, interkulturelle Kommunikation oder Handlungsfähigkeit bieten also ein weites Feld an Deutungsmöglichkeiten und bereiten allein schon aufgrund der oftmals nicht einheitlichen terminologischen Verwendung Probleme (vgl. Krumm 2003: 138). ${ }^{3}$ An dieser begrifflichen Vagheit entzündet sich die Frage, was interkulturelle Handlungsfähigkeit eigentlich bezeichnet. Die Suche nach einer Antwort lässt die Beteiligten oftmals ratlos zurück: „Was genau ist interkulturelle Kompetenz?“, fragen sich die Autorinnen des Handbuches interkulturelle Didaktik und geben eine Kurzdefinition: „Interkulturelle Kompetenz ist die in einem Lernprozess erreichte Fähigkeit, im mittelbaren oder unmittelbaren Umgang mit Mitgliedern anderer Kulturen einen möglichst hohen Grad an Verständigung und Verstehen zu erzielen" (Bertels/Busmann 2013: 33). Diese Publikation des Vereins Ethnologie in Schule und Erwachsenenbildung (ESE) geht damit von einer recht pragmatischen Perspektive aus, setzt die Praktikabilität der Forderungen im Alltag zentral, setzt Verständigung vor Verstehen. Betrachten wir die komplexe Diskussion, die sich um den Terminus Interkulturalität herum entwickelt hat, erhebt sich die Frage, worauf sich die zuständigen Stellen definitorisch g eeinigt haben, um interkulturelle Handlungsfähigkeit als oberstes Lernziel für Fremdsprachen zu postulieren, da diese über das Erlernen von Fremdsprachen offenbar weit hinausgeht. Folgt man der Diskussion um Interkulturalität, bewegen wir uns sofort in einem Bereich der kulturwissenschaftlichen und philosophischen Diskussion, die den Begriff Interkulturalität denen der Multikulturalität und Transkulturalität gegenüberstellt und damit unterschiedliche Wertungen im Umgang der Kulturen untereinander postuliert. Im Zuge der postmodernen und postkolonialen Theorieentwicklung, in der es vor allem um das Aufbrechen gewohnter Denkmuster geht, sind diese Begriffe viel diskutiert worden. Sie

\footnotetext{
${ }^{2}$ Wir wollen an dieser Stelle nicht weiter auf gegenteilige Tendenzen der Globalisierung eingehen, die auf der sichtbaren Oberfläche durchaus Vereinheitlichungsbestrebungen erkennen lassen, so z.B. die „kulturelle“ Variante der „Ikeaisierung“, die unser Wohnverhalten weltweit vereinheitlicht oder die Coffeeshops, die unser Essverhalten steuern. Vgl. hierzu auch Bredella (2012: 77f.).

${ }^{3}$ Vgl. hierzu auch die Lehrwerkdiskussion um „eine Art ,Curriculum‘ für die Entwicklung von interkultureller Kompetenz [, da] interkulturelle Kompetenz immer wieder mit Landeskunde gleichgesetzt wird“ (Meißner/Bär 2007: 127) und demzufolge interkulturelle Kompetenzen nur unzureichend in den Lehrwerken thematisiert werden.
} 
bezeichnen philosophische Konzepte, die mit dem Umgang des Fremden zu tun haben und bergen Ansätze, an denen sich die Geister hinsichtlich des Umgangs mit Kulturen scheiden. Differenz- und Diversitätshypothesen (vgl. Krumm 2003: 139) beleben diese Diskussion um die grundsätzliche Frage, wie Kulturen im Kontext des Eigenen und Fremden zu verstehen sind. Sie unterscheiden zwischen der Vorstellung von zwar gleichwertigen, aber strikt voneinander getrennten Kulturen im multikulturellen Nebeneinander und transkulturellen Ansätzen, denen eine „kulturelle dialogisierende Haltung“ (Thiem 2003: 35) eigen ist, die ein permanentes Aushandeln von Kulturen zur Folge hat. Das Präfix trans- weist dabei auf einen nomadischen Charakter der kulturellen Begegnung $\mathrm{hin}^{4}$, das heißt, kulturelle Differenzen werden zu einem sich bewegenden Netzwerk, in dem sie wie Nomaden von Ort zu Ort ziehen, und so eindeutige Sinnkonstitutionen nicht mehr erlauben. ${ }^{5}$ Während Charles Taylors Versuch der Bestimmung einer „Politik des Multikulturalismus“ (Taylor 1997: 13) zusätzlich den Aspekt der Anerkennung ins Spiel bringt, der jeder Einzelne bedarf und der kulturelle Grenzziehungen noch verfestigt, ist transkulturellen Perspektiven eine ständige Bewegung im Umgang mit kulturellen Besonderheiten eigen, wodurch Grenzziehungen vermieden werden.

In der Abwägung zwischen Trans- und Interkulturalität wird dem Begriff Transkulturalität der Vorwurf gemacht, er bewege sich „außerhalb oder oberhalb eines Bereichs“ (Bredella 2012: 81), während Interkulturalität einen direkten Kontakt mit dem "Gegenüber" (ibid.) ermögliche. Beide Begriffe müssen sich jedoch nicht ausschließen. Während wir zunächst ein Gegenüber haben, mit dem wir in Dialog treten, beginnt ein Prozess der Bewusstwerdung, der (Er)Kenntnis, die in einem immerwährenden Prozess kulturelle Übergänge ermöglicht und Differenzerfahrungen aushandelt. Die schwierige Gratwanderung in der Diskussion um Ab- und Ausgrenzungen, einheitliche Kulturbegriffe, hybride Existenzen oder performative kulturelle Prozesse, offenbart in vielen Texten die moralischen Dilemmata, denen diese Diskussion unterliegt. Sie oszilliert zwischen dem Bemühen, alle Kulturen gleichermaßen anerkennen zu wollen und kultureller Abgrenzung, die zugleich die Furcht birgt, welche das Fremde auslösen kann, weil es uns aus unseren gewohnten Denkmustern herauskatapultiert. ${ }^{6}$

Aber Fremdverstehen bezieht das Fremde in die Auseinandersetzung mit dem Eigenen ein. Dennoch ist Die Frage des Anderen, der Alterität, die sich nicht nur Homi K. Bhabha gestellt hat, und der im Konzept der „Festgestelltheit“ in der ideologischen Konstruktion des „Andersseins“ die Grundlage von Stereotypen

\footnotetext{
${ }^{4}$ Vgl. hierzu Alfonso de Toro (2007: 25).

${ }^{5}$ Vgl. hierzu Thiem (2003: 24ff.) sowie die Theorien zu Differenz und Rhizom bei Gilles Deleuze (1992). Differenz und Wiederholung. München: Fink und Gilles Deleuze/Félix Guattari (1997). Rhizom. Berlin: Merve. Der aus der Botanik stammende Terminus Rhizom, der einen Wurzelstock meint, in dessen Verästelungen Wurzeln und Trieb nicht mehr voneinander unterscheidbar sind, die aber in Verbindung mit der Umwelt stehen, bezeichnet dabei eine erweiterte Konzeption von Differenzen. Vgl. ebenso Wolfgang Welsch (1992 und 1997).

${ }^{6}$ Vgl. hierzu u.a. die Debatte zwischen Clifford Geertz und Richard Rorty, die sich gut kommentiert findet bei Ellrich (1999).
} 
und der Weiterverbreitung von stereotypen Diskursen sieht (Bhabha 2000: 97), nicht das gleiche, wie das Fremde. Fremde sind wir uns selbst, könnte man mit Julia Kristeva (1990) einwenden und doch beinhaltet das Fremde ein Spektrum negativer Konnotationen der Ausgrenzung, die das Andere, die Alterität aus der Perspektive des eigenen kulturellen Vorverständnisses versteht. ${ }^{7}$ Damit stehen das Eigene und das Fremde in einer Wechselbeziehung, die sich angesichts der „höchst unterschiedlichen sprachlich-kulturellen Vorerfahrung“ (Gogolin 2003: 100) der heutigen Gesellschaften vervielfältigt. Alteritätsund Differenzerfahrungen sind Bestandteil unserer aktuellen Gesellschaft und führen dazu, Einheit suggerierende Terminologien aufzugeben, die bislang als nationale identitätsstiftende Merkmale gelten konnten: Nicht mehr Nationalsprache, sondern Verkehrssprache, nicht mehr Land, Nation oder Staat, sondern Territorium ist das neue Orientierungsmodell (ibid.: 101).

Wenn wir Alteritätserfahrungen täglich ausgesetzt sind, was bedeutet dies für den Fremdsprachenunterricht? Ist das Fremde am Fremdsprachenunterricht wirklich fremd oder nur anders im Sinne von unterschiedlich? Und welche Aufgabe hat das Erlernen interkultureller Kompetenzen? Deutlich geworden ist, dass die oben genannte Frage, „was ist interkulturelle Kompetenz“, berechtigt ist. Der Begriff Interkulturalität in seinem kulturübergreifenden Verständnis verweigert sich aber nicht nur einer exakten Definition, er eröffnet uns als Forschungsparadigma durchaus die Möglichkeit, Differenzerfahrung und Alterität in das Bewusstsein zu heben und fruchtbar zu machen:

With the notion of cultural difference, I try to place myself in that position of liminality, in that productive space of the construction of culture as difference, in the spirit of alterity or otherness. (Bhabha in Rutherford1990: 209).

Und das führt uns zum nächsten Schritt, der Klärung des Terminus interkultureller Kompetenz.

\section{2}

Versuchen wir nun, die Begriffe für unser Verständnis von interkultureller Kompetenz einzugrenzen. Wir wollen im Bereich des Fremdsprachenerlernens von einem Kulturbegriff ausgehen, der rein beschreibend und nicht hierarchisierend oder wertend „das Brauchtum, die Sitten, die Manieren, [... ] kurzum alle Eigenarten und Besonderheiten“ (Hansen 2003: 13) eines „Volkes“ meint. ${ }^{8}$ Gesellschaftliche Heterogenität im Sinne einer „unterschiedlichen sprachlichkulturellen Vorerfahrung" (Gogolin 2003: 100) erweitert dabei das Spektrum der kulturellen Eigenheiten und den ambiguen Begriff des Volkes. Dies betrifft

\footnotetext{
${ }^{7}$ Die Verbindung von Hermeneutik und Fremdsprachenerwerb drängt sich an dieser Stelle auf, soll aber nicht weiter verfolgt werden. Vgl. hierzu u.a. Lutzker (1996).

${ }^{8}$ Von einem Territorium zu sprechen, wäre im Rahmen des Erlernens der spanischen Sprache(n) und Kultur(en) abwegig.
} 
die Ausgangskultur(en) ebenso wie die Zielkultur(en). Das Aufbrechen einer einheitlichen Kulturperspektive sowohl der eigenen als auch der fremden Kultur(en) ist damit quasi Voraussetzung für einen interkulturellen Umgang. Das Präfix inter- „orientiert sich am Gegenüber und am Dialog“" (Bredella 2012: 81) und ermöglicht Interaktion, zwischenmenschliche, zwischenkulturelle Kommunikation. Diese umfasst jedoch nicht nur die sprachliche Ebene, sondern auch auf nonverbaler Ebene vokale Zeichen wie Intonation oder Sprachmelodie und im Bereich der Körpersprache Mimik und Gestik. ${ }^{9}$ Interkulturalität geht zunächst von einem Gegenüber aus, taxiert die Unterschiede, die Grenzen, um sich dann auf die andere Kultur einzulassen und sie verstehen zu lernen. Verstehen wiederum ist „der Versuch, mit dem Auftreten von Irritationen fertig zu werden und ebnet uns den Weg zurück ins Selbstverständliche“ (Schwemmer 1997: 136). Dabei bleibt „Fremdverstehen [ein] dynamischer unabschließbarer Bildungsprozess mit Rückwirkungen auf das eigene Selbstund Weltverständnis“ (Bredella 2007: 11), das wiederum „ein Bewusstsein für das Eigene voraussetzt“ (Imbach 2011: 27). Interkulturelle kommunikative Kompetenzen erlernen, bedeutet demzufolge, in fremdsprachlichen Kontexten sensibel handeln zu können, und meint „das Vermögen [...], mit fremden Kulturen und ihren Angehörigen in adäquater, ihren Wertesystemen und Kommunikationsstilen angemessener Weise zu handeln, mit ihnen $\mathrm{zu}$ kommunizieren und sie zu verstehen" (Lüsebrink 2005: 9). Angemessenes verbales und nonverbales Kommunizieren verlangt allerdings ein entsprechendes Instrumentarium, will man diesem Anspruch gerecht werden. Wie dieses Instrumentarium Schülerinnen und Schülern vermittelt werden kann, sagt der Lehrplan aber nicht, die Verantwortung wird an die Schulen weitergegeben:

Indem sich Kernlehrpläne dieser Generation auf die zentralen fachlichen Kompetenzen beschränken, geben sie den Schulen die Möglichkeit, sich auf diese zu konzentrieren und ihre Beherrschung zu sichern. Die Schulen können dabei entstehende Freiräume zur Vertiefung und Erweiterung der aufgeführten Kompetenzen und damit zu einer schulbezogenen Schwerpunktsetzung nutzen. (Kernlehrplan 2013: 8).

Freiräume und schulbezogene Schwerpunktsetzungen sind grundsätzlich nichts Schlechtes, aber angesichts dieser Veränderungen sollte man sich ernsthaft damit auseinandersetzen, dass es die Lehrerinnen und Lehrer sind, die Schülerinnen und Schülern interkulturelle kommunikative Kompetenzen vermitteln sollen. Wenn jedoch, wie oben angedeutet, nicht deutlich genug ist, was der Begriff Interkulturalität umfasst und welches Kulturverständnis dafür notwendig ist, führt uns das Thema vom Schulalltag zur Lehrerausbildung an den Universitäten. Wenn vorausgesetzt wird, dass Lehrerinnen und Lehrer die Anforderungen des Lehrplans erfüllen sollen, muss die Vorbereitung auf diese Aufgabe hinterfragt werden. Ein Blick auf die Lehrerausbildung und den Universitätsalltag zeigt, dass diese Kompetenzen keinen Platz im

\footnotetext{
${ }^{9}$ Vgl. hierzu das Schema der Kommunikationsaspekte bei Košinár (2009: 23).
} 
Ausbildungssystem der Universitäten haben. Zwar sind Veränderungen in den Studienstrukturen der einzelnen Fächer seit Bologna spürbar geworden, so z.B. in der Erhöhung des fachdidaktischen Anteils und der Schulpraktika, doch dienen diese Veränderungen nicht dem im Lehrplan angesprochenen Ziel der interkulturellen Handlungsfähigkeit, sondern der praktischen Umsetzung von Unterrichtseinheiten. ${ }^{10}$ Die Erhöhung des fachdidaktischen Anteils des Studiums geht darüber hinaus zu Lasten der fachwissenschaftlichen Kenntnisse, die sich nur noch auf einem oberflächlichen Niveau bewegen. Damit wird das Verstehen der Zielkultur, die später unterrichtet werden soll, auf ein Minimum reduziert. Gesellschaftliche und historische Zusammenhänge können kaum noch erkannt und Problemstellungen nur begrenzt hinterfragt werden. Zum Verstehen und zur „effektiven Gestaltung interkultureller Kommunikation gehört [aber] die Fähigkeit zur Kulturanalyse“ (Kiel 1997: 9). Diese setzt voraus, dass Lehrerinnen und Lehrer über ein „sehr spezielles kulturelles Wissen“ (ibid.) verfügen sollten, um diesem Anspruch gerecht werden zu können. Woher soll dieses Wissen aber kommen, wenn dieser Bereich im Studium eingekürzt und das Wissen auf Bachelor-Wissen mit Hilfe der universitären Lehrbücher für die modularisierten Studiengänge nivelliert wird und deren verschulter Charakter hinsichtlich einer vertieften Reflexion über die Zielkultur eigentlich aufhorchen lassen sollte, da das „eigene Selbst- und Weltverständnis“ (Bredella 2007: 11) nicht gefordert wird. Doch nicht nur die Reduzierung des fachwissenschaftlichen Wissens ist eine ernstzunehmende Tendenz, sondern auch die Erhöhung des fachdidaktischen Anteils, die auf den ersten Blick positiv erscheint, bleibt ausschließlich theoretisch orientiert. Unterrichtspraktische Übungen werden nur durch Schulpraktika gewährleistet. Für das Vermitteln interkultureller Kompetenzen ist darüber hinaus jedoch entscheidend, dass neben der Sprachkompetenz ein reflektiertes K ulturverständnis entwickelt werden kann, das es der Lehrerpersönlichkeiterlaubt, die Zielkulturin der Schule so glaubwürdig wie möglich zu vermitteln. Hans-Jürgen Lüsebrink verweist eindeutig darauf, dass interkulturelle Kompetenz nicht nur eine „kognitive Dimension [hat, die] allgemein kulturelles Wissen [und] kulturspezifisches Wissen" (Lüsebrink 2005: 9) umfasst, sondern eine weitere Dimension, die oftmals in der Diskussion nicht berücksichtig wird: Die „affektive Dimension“ (ibid.), über die sich „interkulturelle Sozialkompetenz“ (ibid.) ausbilden lässt, damit wir uns nicht unangemessen gegenüber der Zielkultur verhalten. Das heißt, interkulturelle Kompetenz bedeutet nicht nur, dass es um die Vermittlung kulturspezifischer Besonderheiten geht, vielmehr sind wir emotional involviert, wenn wir im Alltag mit Differenzerfahrungen konfrontiert sind. Und diese affektive Dimension findet i n d er L ehrerausbildung k einen P latz. Wenn Studierende ihr Studium beenden, haben sie in der Regel noch nie etwas von Sprechtraining, nonverbaler Kommunikation oder Körpersprache gehört, obwohl in der Forschung Übungen zum Erwerb interkultureller Kompetenzen diskutiert werden. Diese Übungen verweisen deutlich auf die affektive

\footnotetext{
${ }^{10}$ Schon in den 1970er Jahren gab es Bemühungen, Tendenzen der Fremdsprachenlehrerausbildung im Hinblick auf die spätere Berufspraxis kritisch zu reflektieren (vgl. Krumm 1973).
} 
Dimension. Affektive Lernziele „sollen dazu beitragen, dass sich die Lernenden überhaupt auf interkulturelle Kommunikationssituationen und langfristig auf einen Perspektivwechsel einlassen“ (Grau/Würffel 2003: 312). Die Autorinnen unterscheiden in diesem Zusammenhang vier Übungsformen:

Aufgaben zur Wahrnehmungsschulung [...], die vor allem der Förderung affektiver Lernziele dienen; Aufgaben und Übungen zur Sprachreflexion [...] zum Kulturvergleich [...] und zur Entwicklung einer kommunikativen Kompetenz in interkulturellen Kontaktsituationen. (ibid.).

Veränderte Lehreranforderungen sollten auch an der Universität zu veränderten Lehr- und Lernmethoden führen und der universitäre Bildungsprozess dahingehend erweitert werden, die Reflexion und die Erlebbarkeit der Zielkultur(en) gleichermaßen zu gewährleisten. Wie können Studierende also in ihrer Lehrerausbildung gefördert werden, damit das Lehrplanziel der interkulturellen Handlungsfähigkeit nicht mit dem Eintritt in das Schulsystem ad absurdum geführt wird?

Die universitäre Lehrerbildung für das Fach Spanisch ist keine handlungsorientierte und ganzheitliche Ausbildung. Sie setzt sich aus vier Bausteinen zusammen: Fachwissenschaftliche, fachdidaktische und sprachpraktische Kompetenzen werden rein theoretisch vermittelt. Schulpraktische Erfahrungen sind zwar Bestandteil der Curricula, werden aber in die Verantwortung der Schulen gegeben und haben demzufolge mit dem universitären Alltag nur wenig zu tun - abgesehen von den damit verbundenen Verwaltungsaufgaben. Studienbestandteile, die sich mit dem Bereich der Sprecherziehung, der Wahrnehmungsschulung und / oder alternativen Lehrmethoden befassen, sind in der Ausbildung für das Lehramt Spanisch nicht vorgesehen. ${ }^{11}$ Dies führt dazu, dass heutzutage immer noch eine Lücke zwischen den Anforderungen in der Schule und dem Universitätsalltag klafft. ${ }^{12}$ Angesichts der zunehmenden Umstrukturierungen und Anforderungen im Lehrerberuf ist es also notwendig, darüber nachzudenken, einen ganzheitlichen Lehransatz zu verfolgen. Das Konzept der Ganzheitlichkeit meint, „[...] daß der Mensch drei Seiten in seinem Wesen hat. [... ] Leib, Seele und Geist" (Steiner 1988: 147), und wir finden diese Idee bei Lüsebrink wieder, d er z wischen kognitiv-intellektuellen und affektiv-emotionalen Dimensionen unterscheidet und damit versucht, Körper, Geist und Seele miteinander zu verbinden. Solang interkulturelle Kompetenzen nur theoretisch vermittelt werden (wenn sie überhaupt als

\footnotetext{
11 Forschungen zu Lehr- und Lernmethoden im weitesten Sinne dramapädagogischer Ansätze finden sich v.a. für d ie F ächer Anglistik / A merikanistik und D eutsch als Fremdsprache (DaF). Für die Vermittlung der spanischen Sprache stecken die Forschungen noch in den Kinderschuhen.

12 Man könnte diese Kette noch fortsetzen, indem man nach den Kompetenzen der Hochschullehrerinnen und lehrer fragt, doch das wäre ein weiterer Schwerpunkt.
} 
Kompetenz eine Rolle im universitären Alltag spielen), wird sich die Kette der Unvereinbarkeiten von Ausbildungsinhalten und praktischem Schulalltag fortsetzen und Auswirkungen auf den Unterricht haben:

Eine Bildungs- und Erziehungsmethode, die ausschließlich auf dem Anhäufen von Wissen und der Entwicklung des rationalen, kausalen Denkens beruht und der Entwicklung des Empfindens und Fühlens keine Aufmerksamkeit schenkt, führt später zu Erwachsenen, für die eine Harmonie zwischen dem Denken und dem Fühlen unnatürlich, wenn nicht unmöglich ist. Die gleichzeitige Entwicklung der sensorischen und der logischen Bereiche ist unerläßlich für die Entwicklung des Gleichgewichts und der Harmonie des ganzen Menschen. (Lutzker 1996: 265).

Die im Kernlehrplan angelegte interkulturelle kommunikative Kompetenz umfasst die fünf Merkmale Verstehen, Handeln, Wissen, Einstellungen und Bewusstheit. Handeln können wir aber erst, wenn wir verstehen und verstehen können wir erst, wenn wir wissen oder uns bestimmte Aspekte bewusst werden. Handeln als sprachliche Interaktion bezeichnet den direkten Kontakt mit dem Gegenüber in möglichst unverfälschten und glaubwürdigen Situationen. Doch darf darüber der Körper als wichtiges Kommunikationsmedium nicht vergessen werden. Universitäre Bildung kann, ohne die Strukturen großartig verändern $\mathrm{zu}$ müssen, relativ unproblematisch dazu beitragen, handlungsorientierte, körperbetonte Lehrkonzepte auf der Basis performativer Ansätze zu integrieren. Der Begriff des Performativen orientiert sich dabei zunächst an John L. Austins Sprechakttheorie. Für die Umsetzung performativ basierter Unterrichtsanteile an der Universität ist die Bedeutung des Verbs „to perform“ interessant, auf die Austin in seiner Sprechakttheorie verweist und die das Vollziehen von Handlungen meint (vgl. Austin 1979: 29). Erika Fischer-Lichte überträgt in ihrer Ästhetik des Performativen den Begriff der Handlungen auf die körperliche Ebene, denn „eine solche Anwendung drängt sich geradezu auf" (FischerLichte 2004: 34). Vor dem Hintergrund, dass Handlungen Wirklichkeit(en) konstituieren, hebt sie hervor, dass „körperliche Handlungen, die als performativ bezeichnet werden [...] keine vorgängig gegebene Identität zum Ausdruck [bringen, sondern] Identität [...] allererst hervor[bringen]“ (Fischer-Lichte 2004: 37). Der hier Anwendung findende Begriff performance geht auf Judith Butlers Performative Acts zurück, die Geschlechtsidentitäten (sex/gender) hinterfragt. ${ }^{13}$ Performance ist ,aber nicht im Sinne einer bewußt theatralischen Selbstinszenierung [zu verstehen], sondern als unbewußte Matrix, die sich aus der ständigen Re-Inszenierung vorgegebener Normen ergibt" (Thiem 2003: 213). Diese Grundidee können wir für einen performativ orientierten Unterricht nutzbar machen, denn sie erlaubt es, Alterität in unterschiedlicher Form kognitiv und körperlich / affektiv, also ganzheitlich zu erfahren. Der Kontakt zwischen den Kulturen führt dabei zu einem dynamischen und reflexiven Prozess, in dem Identitäten und Differenzen verhandelbar werden, ohne dabei im Umkehrschluss festgelegte Identitäten zu begründen. Die oben genannten

\footnotetext{
13 Vgl. Judith Butler (1988).
} 
Übungen zum Erwerb interkultureller Kompetenzen im universitären Alltag liegen vor allem im Bereich der Sprachreflexion und in Teilen im Kulturvergleich. Die Praxis zeigt, dass Studierende keine Erfahrung mit Wahrnehmungsschulung und performativen Lehrmethoden haben, die über weit zurückliegende TheaterAGs in der Schulzeit hinausreichen. Sie können im Lernprozess nur selten Körper, Stimme und Präsenz als ein Ganzes wahrnehmen. Wahrnehmung bedeutet, Bewusstheit entwickeln gegenüber dem eigenen Körper, den Zusammenhang von Stimme, Atmung und Gefühl als Ausdruck des eigenen Körpers und der eigenen Präsenz erfahren, als Tatsache des Ich-bin-in-der-Welt. Die Universität ist nicht der Ort, um mit allen Sinnen zu lernen und doch kann uns ein oftmals unbeliebter Unterrichts- bzw. Studienbestandteil dabei helfen, auf diesem Weg ein Stück voranzuschreiten. Literatur. Wer kennt sie nicht, die Lustlosigkeit der Studierenden angesichts einer Lektüreaufforderung, die sich bis zur Verweigerung oder Dozentenschelte steigern kann. Lesen, heißt es aus Studierendenmunde, gehöre heutzutage immer weniger zum alltäglichen Beschäftigungsspektrum. Der Buchmarkt zeigt ein anderes Bild. Auf die Frage, warum lesen so viel Unlust verursacht, ist immer wieder zu hören, dass Studierende mit Literatur gar nichts anfangen können, da die Texte viel zu lang, zu kompliziert oder zu langweilig seien. Bei näherer Rückfrage stellt sich allerdings schnell heraus, dass die Unlust in den meisten Fällen das Resultat der Schulbildung ist und oftmals mit der Beschneidung von Kreativität verbunden wird. Das heißt, Studierende kritisieren rückblickend am Schulunterricht, dass sie meistens der Interpretation der Lehrenden folgen mussten und kaum Raum für eigene Gedanken hatten. Erstaunt reagieren Studierende darauf, dass es im Grunde keine festgelegte Interpretation gibt und Sinn sich erst langsam über einen Reflexions- und Wahrnehmungsprozess konstituiert. Den geringen Stellenwert von Literatur finden wir auch im Lehrplan seit jeher terminologisch besiegelt: "Ganzschriften“ ist dort zu lesen als übliche Umschreibung für Romane, Theaterstücke oder Gedichte, die in voller Länge gelesen werden sollten. Die mitschwingende pejorative Konnotation in diesem Begriff macht die mangelnde Wertschätzung deutlich und mindert das Erstaunen über den unmotivierten Zugang der Studierenden zur Literatur. Dass Lesen auch in den PISA-Studien ausschließlich auf das Lesen von Sachtexten beschränkt bleibt und Literatur als integrativer Bestandteil für die Persönlichkeitsentwicklung gering geschätzt wird, untermauert das Gesagte. Lothar Bredella führt an, dass es für PISA nur um einen „funktionalistischen Ansatz“ (Bredella 2007: 30) gehe, der im Kontext der späteren Berufsausübung zu sehen sei. Hierzu sei die Anmerkung erlaubt, dass im Rahmen dieser berufsorientierten Zukunftsperspektive der Lehrerberuf als zukünftiges Berufsfeld für PISA nur wenig Bedeutung zu haben scheint.

\section{4}

Der „Bildungssinn literarischer Texte“ (ibid.) ist nicht eben eine neue Errungenschaft unserer Zeit. Über die ästhetische Erziehung des Menschen wusste schon 
Friedrich Schiller zu philosophieren. Dass sich in der Literaturwissenschaft im Laufe des 20. Jahrhunderts Tendenzen herausgebildet haben, die das Interesse am Bildungssinn von Literatur zugunsten einer formalen Analyse von Texten verlagert haben, soll hier nicht das Thema sein. Es geht vielmehr darum, zu zeigen, dass Literatur trotz aller Anfeindungen das Potential in sich trägt, Wahrnehmung zu stärken - und damit greifen wir auf die Bedeutung des Begriffes Aisthesis im Sinne von Wahrnehmung zurück. Literatur kann Wirklichkeit oder Welt konstituieren und Wirklichkeitserfahrungen über den Text erfahrbar machen. Die „Auseinandersetzung mit Lebensentwürfen und gesellschaftlichen Verhältnissen geschieht [dabei] nicht nur auf einer kognitiv-analytischen Ebene, sondern auch auf einer sinnlich-anschaulichen" (Eilers-Bierbaum 2004: 41), vor allem dann, wenn Literatur in Handlung übertragen wird. Gerade im Bereich des Fremdsprachenerwerbs öffnet Literatur das Tor zum Umgang mit Alterität. Wenn von „Empathie, Perspektivenübernahme und Fremdverstehen“ (Nünning/Surkamp 2013: 157) als Lernzielen eines interkulturellen Literaturunterrichts die Rede ist, kann Empathie als „Form der Fremderfahrung“ (Baring 2011: 23) verstanden werden, die es ermöglicht, „sich in Andere und Anderes einzufühlen" (Curtis 2009: 12). In einem kognitiven Prozess wird aber dabei nicht nur das Beobachtete erlebt, sondern darüber hinaus zusätzlich das Verstehen des Verhaltens erreicht. ${ }^{14}$ Mit Literatur lässt sich zudem eine ästhetische Bildung erreichen, die ganz im humanistischen Sinne Körper, Geist und Seele in ein harmonisches Miteinander zu versetzen mag. Literatur eröffnet Raum, interkulturelle Begegnungen handlungsorientiert zu erfahren, sie ist quasi Treffpunkt gelebter Kultur(en). Die Aufwertung von Literatur kann zu einem ganzheitlichen Lehr-/Lernkonzept beitragen, das sich mit ein wenig Engagement durchaus im Studienalltag durchsetzen lässt:

Literarische Texte werden demnach dadurch bedeutsam für den Unterricht, dass sie den Leser durch ihre poetische Unbestimmtheit zur kreativen Mitwirkung an der Sinnkonstitution herausfordern, sein lebensweltliches Vorverständnis aktivieren und zur Kommunikation anregen. (Nünning/Surkamp 2013: 149).

Im Kernlehrplan wird Literatur jedoch ausschließlich dem Punkt Text- und Medienkompetenz zugeordnet. Schülerinnen und Schüler sollen

sich mit den Perspektiven und Handlungsmustern von Akteuren, Charakteren und Figuren auseinandersetzen, ggf. einen Perspektivwechsel vollziehen [...] ihr Textverständnis durch das Verfassen eigener produktionsorientierter, kreativer Texte zum Ausdruck bringen [... ]. (Kernlehrplan 2013: 23).

Der „analytisch-interpretierende[...] sowie [...] produktions- bzw. anwendungsorientierte[...] Umgang“ (ibid.) mit Texten sieht Textproduktion als rein

\footnotetext{
${ }^{14}$ Vgl. hierzu die Unterscheidung von „basic“ und „reactive empathy“ bei Karsten R. Stueber (2006). Rediscovering Empathy. Agency, Folk Psychology, and the Human Sciences. Cambridge: MIT Press.
} 
schriftlichen Ansatz, zieht Körperbewusstsein nicht in Betracht. Den Körper stärker zu akzentuieren, wäre jedoch wünschenswert. In der Dramapädagogik wird dies „mit einer neuen, die Körperlichkeit stark akzentuierenden Lehr/Lernkultur" (Schewe 2011: 22) erfolgreich umgesetzt. Warum können Anteile davon nicht auch in den regulären Studienalltag Eingang finden? Die Welt und sich selbst wahrnehmen und in einem performativen Akt gestalten, bedeutet zugleich eine veränderte Rolle der Lehrperson, die nicht mehr allein für den Fortgang des Unterrichts verantwortlich ist. Sowohl Studierende als auch Lehrende partizipieren gemeinsam am Prozess des Erwerbs interkultureller Handlungsfähigkeit. Ausgehend von der Vorstellung eines gemeinsam erlebten Prozesses, habe ich selbst verstärkt Lehransätze in den Unterricht einfließen lassen, die Körper und Körperlichkeit beim Erlernen von Fremdsprachen und Kulturen einen zentralen Stellenwert zukommen ließen. Der Weg führte dabei zunächst über die Umsetzung dramatischer Texte in (Theater)Spiel. Kleine Einschübe des szenischen Interpretierens von Textstellen aus bestimmten Theaterstücken in literaturwissenschaftlichen Seminaren zeigten erste Erfolge im Hinblick auf den Zugang zu dramatischen Texten und die Merkfähigkeit sprachlicher und kultureller Besonderheiten. Lyrik-Performances erweiterten das Feld der Textgattungen und trugen zum Erleben von Gefühlen, aber auch von heute selten erlebter Stille bzw. Schweigen bei, die gerade in der Lyrik von besonderer Relevanz ist und körperliches Bewusstsein noch stärker herausfordert. ${ }^{15}$ Performative Lehr- und Lernmethoden bieten eine geeignete Basis, um über Literatur und Theater verschiedene Kompetenzen spielerisch zu entwickeln. Ästhetische Bildung funktioniert über körperliches Lernen, denn in diesem Prozess des Wahrnehmens wird Wissen im wahrsten Sinne des Wortes verkörpert. Wir lernen, indem wir Text in Körper umsetzen, denn so bleibt das Wissen stärker in der Erinnerung verhaftet. Die Möglichkeit, über Literatur und Theater neue, fremde Welten zu konstruieren, macht Lernen zu einer kreativen, persönlichen und körperbezogenen Erfahrung, die nicht nur interkulturelles Miteinander gewährleistet, sondern auch soziale Kompetenzen erfordert und durch ein neues Körperbewusstsein Fortschritte in der Persönlichkeitsentwicklung forciert. Das Spektrum neugewonnener Erfahrung und die positiven Auswirkungen dieser ersten Schritte hin zu einer veränderten Lernkultur wurden zusätzlich in Kursen zur nonverbalen Kommunikation und Körpersprache umgesetzt, die das theoretische Wissen der Fachdidaktik-Kurse ergänzten. Ermutigt durch die positiven Rückmeldungen, habe ich mich entschlossen, mit Studierenden Theaterstücke zu inszenieren und sie auf der universitätseigenen Theaterbühne $\mathrm{zu}$ präsentieren. Dies stellt jedoch eine große Herausforderung für alle Beteiligten dar und erfordert ein hohes Maß an Engagement, da es zeitlich die Pflichtveranstaltungen bei weitem überschreitet.

\footnotetext{
15 Vgl. Thiem (2013). Der Aufsatz Connotaciones del silencio versucht zu verdeutlichen, dass der Begriff silencio, der in der spanischen Sprache nicht die Differenzierung von Stille und Schweigen erlaubt, durchaus unterschiedliche Konnotationen birgt, die über die genannten Bedeutungen hinausgeht. Dies wiederum kann für eine gesteigerte Aufmerksamkeit im Bereich der Körperarbeit fruchtbar gemacht werden.
} 
Nur über die freiwillige Teilnahme ist es möglich, Theater in die Lebenswelt der Studierenden zu bringen. Die seit 2008 an der Universität Paderborn jährlich stattfindenden Theateraufführungen in spanischer S prache stellen in jeder Produktion neue Herausforderungen an alle Mitwirkenden. Begonnen haben wir ${ }^{16}$ mit ersten kurzen Theaterstücken und haben uns zugleich mit den ersten Schwierigkeiten konfrontiert gesehen. Wir stellten fest, dass sich vorwiegend Studentinnen für das Theaterspiel fanden. Wie immer, wenn es sich um das Verlassen der rein kognitiven Ebene handelt, zieht sich das männliche Geschlecht zunächst zurück. Wir haben die fehlenden männlichen Darsteller durch weibliche ersetzt und diese Situation hatte durchaus etwas für sich. Das Hineinschlüpfen in Rollen des entgegengesetzten Geschlechts, das cross gender, führte zu einer erhöhten Bewusstwerdung in diesem Fall männlicher Verhaltensgewohnheiten wie Gangart, Sprache, Gestik und Mimik nicht nur bei den jungen Frauen, sondern auch bei ihren männlichen Kommilitonen. Die Kostüme erlaubten eine Überstilisierung der männlichen Attribute, trugen zu einer Reflexion der Geschlechterkonstruktionen ebenso bei wie zu einer neuen Wahrnehmung des eigenen Geschlechts und halfen, ein großes Spektrum sozial konstruierter Männlichkeitsbilder erkennen zu lernen. Nach den ersten Erfolgen haben wir uns schließlich an größere Theaterstücke gewagt. Mit der Größe der Stücke nahm auch die Zahl der männlichen Teilnehmer zu, da sie von uns direkt angeworben wurden. Der Arbeitsaufwand für die großen Produktionen ist erheblich höher und fordert aufgrund der hohen Anzahl an Protagonisten nicht nur interkulturelle, sondern vor allem soziale Kompetenzen wie Teamfähigkeit, Kooperationsbereitschaft, Konfliktbewältigung o der Kritikfähigkeit von den Studierenden. Viele Studierende, von denen viele in Deutschland aufgewachsen sind, aber unterschiedliche kulturelle Erfahrungen mitbringen, bescheren in jeder Produktion ein heterogenes Gruppenprofil. Türkische, spanische und lateinamerikanische, osteuropäische und deutsche Kulturerfahrungen treffen dabei aufeinander, um sich über eine gemeinsame fremdsprachliche Zielkultur auszutauschen. Eine große (Theater)Gruppe bedeutet zugleich viele unterschiedliche Ansichten, viele verschiedene Charaktere. Allen gemeinsam aber ist zu Beginn des Projektes immer die mangelnde körperliche Selbstwahrnehmung, und das ist über die verschiedenen Kulturen hinweg einheitlich. Allen Aufführungen voran geht daher ein Prozess des Erlernens bestimmter Übungen zur Atmung, Wahrnehmungsschulung, Körpersprache, Artikulation und Intonation. Diese Übungen dienen dazu, Körperlichkeit und Sprache bewusster zu erleben und die Präsenz auf der Bühne erfahrbar zu machen. ${ }^{17}$ Das Schwierigste bei den Produktionen war es, Gruppengefühl, Teamwork, Ausdauer und Disziplin zu erreichen und aufrechtzuerhalten. Da jede Produktion ein ganzes Jahr in Anspruch nimmt, ist vor allem die Ausdauer ein oftmals schier

\footnotetext{
${ }^{16}$ Die ersten Aufführungen habe ich in Zusammenarbeit mit meiner Kollegin Antonieta Sánchez-Llorente betreut.

17 Die Übungen lassen sich recht gut nachvollziehen u.a. bei Malte Pfeifer/Volker Liste. (2009). Kursbuch Darstellendes Spiel. Stuttgart: Klett oder Maike Plath. (2009). Biografisches Theater in der Schule. Weinheim/Basel: Beltz. Darüber hinaus gibt es eine Reihe weiterer Übungsbücher, die man für den Fremdsprachenerwerb kreativ umgestalten kann.
} 
unlösbares Problem. Aber auch die Motivation der Einzelnen variiert sehr stark, denn man arbeitet mit Studierenden, von denen einige kein Selbstvertrauen besitzen, andere dafür umso mehr, Studierende, die Lampenfieber haben oder Bühnenpräsenz lieben oder viele weitere Ängste und Unsicherheiten in sich tragen. So kann der Entwicklungsprozess jeder/s Einzelnen fast zu einer Art persönlicher Therapie werden, indem bestimmte Persönlichkeitsmerkmale eine erhöhte Aufmerksamkeit erfahren.

Die einzelnen Aufführungen haben unterschiedliche Erfahrungen bereitgehalten. So wurde in der Aufführung von La casa de Bernarda Alba von Federico García Lorca - ein Stück, das nur aus Darstellerinnen besteht - auch die ästhetische Dimension des Theaterspielens sichtbar, indem in einer der ersten Szenen alle Frauen gleichzeitig ihren Fächer öffnen sollten. Hierbei war es wichtig, dass ein einheitliches Bild entstand und es erforderte ein ungeheures Maß an Disziplin und Körperbewusstsein, damit dieses Bild seine Wirkung entfalten konnte. Körperpräsenz war an dieser Stelle das Medium der Stimmungsvermittlung. Weitaus stärkere körperliche Präsenz mussten Studierende beiderlei Geschlechts in dem Drama von Fermín Cabal Tejas verdes zeigen. Da es sich hier um eine in Monologform gestaltete Auseinandersetzung mit der chilenischen Diktatur handelt, mussten alle sechs Darstellende den gesamten Aufführungszeitraum auf der Bühne verbringen. Konzentration über den gesamten Spielverlauf hat die Studierenden bis an ihre körperlichen Grenzen gebracht (Rückenschmerzen, eingeschlafene Gliedmaßen, etc.) und Disziplin und Geduld eingefordert. Die nachfolgende Produktion Monólogos tangueros war ein selbst erstelltes Theaterspiel zum Thema Tango. Eine Collage aus Literatur, Musik, Gesang und Tanz hat alle Studierenden zu einem Teil der Produktion des Stückes und zugleich zum Interpreten werden lassen. Vor allem Gesang und Tanz waren für die meisten Neuland. In zusätzlichen Tanzworkshops konnten sie sich diese Fähigkeiten aneignen, um sie auf der Bühne zu präsentieren. Die Kombination von Sprache, selbst gespielter Musik sowie selbst interpretiertem Tanz und Gesang hat diese Inszenierung zu einer der produktivsten und gruppendynamischsten Prozesse überhaupt werden lassen. Das Thema hat die Studierenden zudem mit stereotypen Verhaltensmustern konfrontiert, die dem Tango eigen sind: Männliche Dominanz und weibliche Unterwürfigkeit. So konnten sie zugleich interkulturell bezogene Erfahrungen machen, denen die meisten in ihrer gewohnten Umgebung in dieser Form noch nicht begegnet waren. Auch die Inszenierung des spanischen Klassikers Don Juan (El burlador de Sevilla y convidado de piedra) von Tirso de Molina hat weitere Erfahrungsmomente bereitgehalten. Hier ging es vor allem darum, stereotype Bilder eines heißblütigen spanischen „Don Juan“ zu überwinden. Alle Beteiligten mussten sich damit auseinandersetzen, dass Stereotypenbildung oftmals den Denkprozess erheblich einschränkt. In diesem Stück mussten zudem einige Rollen umbesetzt werden, da die Darsteller den verschiedenen Anforderungen der Rolle nicht gewachsen waren. Diese Be- und Umbesetzungen, die dem Funktionieren der Aufführung geschuldet waren, brachten Eifersucht, Neid, 
Tränen und Enttäuschung mit sich. Um diese Situation zu retten, gab es Einzelund Gruppengespräche mit und ohne meine Anwesenheit. Die Erfahrungen wurden schließlich in Erfahrungsberichten noch einmal schriftlich verarbeitet. Im Nachhinein konnten auch diese Erfahrungen positiv gewertet wurden, da sie den Studierenden klar gemacht hatten, dass in diesen großen Produktionen Teamwork die wichtigste Kompetenz für das Gelingen der Aufführung ist. Es wurde deutlich, dass man auch in einer anderen Rolle als der gewünschten eine gute Position einnehmen kann und dass es nicht immer nur die großen Rollen sind, die zum Gelingen des Stückes beitragen.

Im Rückblick auf die vergangenen Produktionen und die damit verbundenen unterschiedlichen und oftmals nicht vorhersehbaren, aber meistens positiven Erfahrungen lässt sich feststellen, dass Elemente des Theaterspiels in einen performativ ausgerichteten Unterricht integriert werden können, um gute Resultate im Hinblick auf das Erlernen von interkulturellen kommunikativen Kompetenzen zu erzielen und interkulturelle Handlungsfähigkeit und Persönlichkeitsentwicklung gleichermaßen zu schulen. Insofern sollten performative Lehr-/Lernstrategien Bestandteil eines jeden Studienalltags in der Lehrerbildung werden, um Studierende zu interkulturell handelnden starken Persönlichkeiten herauszubilden. Dabei muss es sich nicht nur um Theateraufführungen handeln, vereinzelte Formen des Darstellendes Spiels oder des Szenischen Interpretierens wären schon gute Ansätze. ${ }^{18}$ Nur wenn Studierende die Chance haben, sich selbst weiterzuentwickeln und dabei die Gesamtheit von Körper, Geist und Seele berücksichtigen, können sie im Schulalltag den Anforderungen des Lehrplans gerecht werden. Performative Lehr-/Lernstrategien ermöglichen den Dialog mit fremden Kulturen auf spielerische Art und Weise, erfordern Teamarbeit und Konfliktlösungspotential, fördern Empathie im Sinne einer notwendigen Alteritätserfahrung, Kreativität im Umgang mit ästhetischer Bildung und Flexibilität im Hinblick auf die Begegnung mit fremden Kulturen, erhöhen die Entscheidungsfähigkeit sowie die Frustrationstoleranz, trainieren unsere kognitiv-analytischen Fähigkeiten ebenso wie unsere emotionalen. Also alles, was wir für das Erlernen interkultureller Kompetenzen benötigen. Unterstützung seitens der Universität gibt es für solche Projekte natürlich nicht. Dass es sich lohnt, dennoch weiter daran zu arbeiten, zeigen die vielen positiven Rückmeldungen der Studierenden, die aus den Seminaren und Aufführungen etwas für sich mitgenommen haben. Ein Student, der in seinem Lehramtsstudium neben Spanisch auch Sport (!) studiert, beschrieb diese Erfahrung so: „Ich danke Ihnen für diesen Kurs, denn mir ist Vieles bewusster geworden. Ich habe gelernt, aufrecht zu gehen und auf meine Körpersprache zu achten“. Ist

\footnotetext{
18 Nünning/Surkamp bezeichnen dies auch als Teaching narrative texts, teaching poems und teaching plays als Anregung für die Unterrichtspraxis (vgl. Nünning/Surkamp 2013). Im Grunde ist es nichts anderes, als Literatur entsprechend ihren gattungsspezifischen Besonderheiten zu unterrichten. Und dies sollte doch eigentlich sowieso das Ziel sein.
} 
es nicht genau das, worauf es im Leben ankommt: Bewusstwerdungsprozesse zu initiieren, um selbst Welt gestalten zu können und „[d]ie Welt auch im fremdsprachlichen Unterricht immer wieder neu [zu] verzaubern" (Schewe 2011: 20).

\section{Bibliografie}

Austin, John L. (1979): Zur Theorie der Sprechakte. 2. Aufl. Stuttgart: Reclam

Baring, Frank (2011): Empathie und historisches Lernen. Frankfurt am Main u.a. / Peter Lang

Bertels, Ursula/Bussmann, Claudia (2013): Handbuch interkulturelle Didaktik. Münster/New York/München/Berlin: Waxmann

Bhabha, Homi K. (2000): Die Verortung der Kultur. Tübingen: Stauffenberg

Bredella, Lothar (2007): „Fremde Welten entwerfen. Der Bildungssinn literarischer Texte“, in: Christiane Fäcke/Wolfgang Wangerin (Hrsg.): Neue Wege zu und mit literarischen Texten. Baltmannsweiler: Schneider Verlag Hohengehren, 30-50

Bredella, Lothar (2012): Narratives und interkulturelles Verstehen. Zur Entwicklung von Empathie-, Urteils- und Kooperationsfähigkeit. Tübingen: Narr Francke Attempto

Budde, Monika (2012): Über Sprache reflektieren. Unterricht in sprachheterogenen Lerngruppen. Kassel: Kassel University Press

Bundesministerium für Bildung und Forschung (2009): Zur Entwicklung nationaler Bildungsstandards. Eine Expertise http://www.bmbf.de/pub/zur_entwicklung_nationaler_bildungsstandards.pdf (Accessed 17 September 2014)

Butler, Judith (1988): „Performative Acts and Gender Constitution: An Essay in Phenomenology and Feminist Theory", in: Theatre Journal, 40, 4: 519-531

Curtis, Robin/Koch, Gertrud (Hrsg.) (2009): Einfühlung. Zur Geschichte und Gegenwart eines ästhetischen Konzepts. München: Fink

Eilers-Bierbaum, Christiane (2004): „Szenisches Interpretieren als Einstieg in die Arbeit mit literarischen Texten"; in: Javier García de María/Ursula Vences (Hrsg): Alles von der Rolle im Spanischunterricht. Berlin: tranvia, 40-62

Ellrich, Lutz (1999): Verschriebene Fremdheit. Frankfurt am Main: Campus

Fischer-Lichte, Erika (2004): Ästhetik des Performativen. Frankfurt am Main: Suhrkamp

Gogolin, Ingrid (2003): „Interkulturelle Erziehung und das Lehren und Lernen fremder Sprachen“, in: Karl-Richard Bausch/Herbert Christ/Hans-Jürgen Krumm (Hrsg.): Handbuch Fremdsprachenunterricht. Tübingen und Basel: Francke, 96-102 
Gogolin, Ingrid/Krüger-Potratz, Marianne (2006): Einführung in die Interkulturelle Pädagogik. Opladen \& Farmington Hills: Verlag Barbara Budrich

Grau, Maike/Würffel, Nicola (2003): „Übungen zur interkulturellen Kommunikation", in: Karl-Richard Bausch/Herbert Christ/Hans-Jürgen Krumm (Hrsg.): Handbuch Fremdsprachenunterricht. Tübingen und Basel: Francke, 312-314

Hansen, Klaus P. (2003): Kultur und Kulturwissenschaft. 3. Aufl. Tübingen: Francke

Imbach, Werner (2011): Zeitgenössisches spanisches Theater im Spanischunterricht. Hamburg: disserta

Kernlernplan für die Sekundarstufe II Gymnasium / Gesamtschule in Nordrhein-Westfalen 2013. Ministerium für Schule und Weiterbildung des Landes Nordrhein-Westfalen. www.schulministerium.nrw.de (Accessed 17 September 2014)

Kiel, Ewald (1997): „Die Entwicklung interkultureller Kommunikationskompetenz aus der Sicht der interkulturellen Didaktik“, in: Wolfgang Börner/Klaus Vogel (Hrsg.): Kulturkontraste im universitären Fremdsprachenunterricht. Bochum: AKS Verlag, 3-20

Košinár, Julia (2009): Körperkompetenzen und Interaktion in pädagogischen Berufen. Bad Heilbrunn: Julius Klinkhardt

Kristeva, Julia (1990): Fremde sind wir uns selbst. Frankfurt am Main: Suhrkamp

Krumm, Hans-Jürgen (1973): Analyse und Training fremdsprachlichen Lehrverhaltens. Weinheim und Basel: Beltz

Krumm, Hans-Jürgen (2003): „Curriculare Aspekte des interkulturellen Lernens und der interkulturellen Kommunikation“, in: Karl-Richard Bausch/Herbert Christ/Hans-Jürgen Krumm (Hrsg.): Handbuch Fremdsprachenunterricht. 4. Aufl. Tübingen und Basel: Francke, 138-144

Lüsebrink, Hans-Jürgen (2005): Interkulturelle Kommunikation: Interaktion, Fremdwahrnehmung, Kulturtransfer. Stuttgart: Metzler

Lutzker, Peter (1996): Der Sprachsinn. Sprachwahrnehmung als Sinnesvorgang. Stuttgart: Verlag Freies Geistesleben

Meißner, Franz-Joseph/Bär, Marcus (2007): „Didaktik des Fremdverstehens / Interkulturelles Lernen in Lehrwerken des Spanischunterrichts", in: Lothar Bredella /Herbert Christ (Hrsg.): Fremdverstehen und interkulturelle Kompetenz. Tübingen: Narr Francke Attempto, 109-132

Nünning, Ansgar/Surkamp, Carola (2013): Text - Literatur - Kultur: Handlungs- und produktionsorientierter Literaturunterricht", in: Gerhard Bach/Johannes-Peter Timm (Hrsg.): Englischunterricht. Grundlagen und Methoden einer handlungsorientierten Unterrichtspraxis. 5 Aufl. Tübingen und Basel: A. Francke, 148-171 
Rutherford, Jonathan (1990): „The Third Space. Interview with Homi Bhabha", in: Jonathan Rutherford (Hrsg.): Identity: Communitiy, Culture, Difference. London: Lawrence and Wishart, 207-221

Schewe, Manfred (2011): „Die Welt auch im fremdsprachlichen Unterricht immer wieder neu verzaubern - Ein Plädoyer für eine performative Lehrund Lernkultur", in: Almut Küppers/ Torben Schmidt/Maik Walter (Hrsg.): Inszenierungen im Fremdsprachenunterricht: Grundlagen, Formen, Perspektiven. Braunschweig: Westermann Schroedel Diesterweg Schoeningh Winklers, 20-31

Schwemmer, Oswald (1997): Die kulturelle Existenz des Menschen. Berlin: Akademie Verlag

Steiner, Rudolf (1988): Einführung in die Anthroposophie. Dornach: Rudolf Steiner Verlag

Taylor, Charles (1997): Multikulturalismus und die Politik der Anerkennung. Frankfurt am Main: Suhrkamp

Thiem, Annegret (2003): Repräsentationsformen von Subjektivität und Identität in zeitgenössischen Texten lateinamerikanischer Autorinnen. Frankfurt am Main: Vervuert

Thiem, Annegret (2013): „Connotaciones del silencio“, in: Christina Bischoff/Annegret Thiem (Hrsg.): Poesía del silencio. Münster: LIT, 201-210

Toro, Alfonso de (2007): "Dispositivos transmediales, representación y anti-representación . Frida Kahlo: Transpictorialidad - Transmedialidad", in: Comunicación, 5: 23-65

Welsch, Wolfgang (1992): "Transkulturalität. Lebensformen nach der Auflösung der Kulturen”, in: Information Philosophie, 2: 5-20

Welsch, Wolfgang (1997): „Transkulturalität. Zur veränderten Verfassung heutiger Kulturen, in: Irmela Schneider/Christian W. Thomson (Hrsg.): Hybridkultur. Medien, Netze, Künste. Köln: Wienand Verlag, 67-90 